\title{
Bar Destruction Mechanism by External Interactions
}

\author{
M. T. Nishida \\ Kobe Women's University, Japan \\ K. Wakamatsu \\ Physics Department, Gifu University, Japan
}

\begin{abstract}
.
To investigate bar destruction by a vertical head-on galaxy encounter, the dynamical evolution after the instantaneous momentum change of the impulsive approximation of a vertical head-on galaxy encounter, and the instability of finite amplitude one-armed perturbations to barred structure, are investigated by 2-dimensional N-body simulations.

Preliminary results show that off-center vertical head-on collisions induce bar destruction but collisions in which the colliding galaxy passes through the very center of the target galaxy do not induce bar destruction.
\end{abstract}

\section{Introduction}

It is well known that a galaxy encounter induces barred structure (Noguchi 1987), but the formed barred structure is not affected so severely by tidal encounters which induce bridges and tails (e.g., Gerin, Combes, \& Athanassoula 1990 ). The perturbing potential in such a galaxy encounter is essentially bisymmetric.

On the other hand, the perturbing potential in the case of a rapid, vertical, off-center, head-on collision between galaxies has quite a different geometry: one-armed symmetry. Stability analyses of barred stellar systems against such perturbations have not been fully studied yet. Thus, the possibility of bar destruction by such a galaxy encounter is not denied.

In this paper, we performed a set of $\mathrm{N}$-body brute-force simulations to study whether barred structures that were formed by so-called bar instabilities can be destroyed by a rapid vertical off-center head-on collision.

\section{Numerical Method}

Before proceeding with full 3-dimensional N-body simulations, we adopted here a 2-dimensional cloud-in-cell N-body code (Miller 1976; Sellwood 1981). The initial mass model is the Toomre-Kuz'min disk with a length scale $a_{d}=10$ grid units and a total mass $\mathrm{M}_{t}$. Bar instability of this disk is followed from $t=0$ to $t=100$ in units of $\left(\mathrm{GM}_{t} / a_{d}{ }^{3}\right)^{-1 / 2}$. Hereafter, time is measured in this unit. 


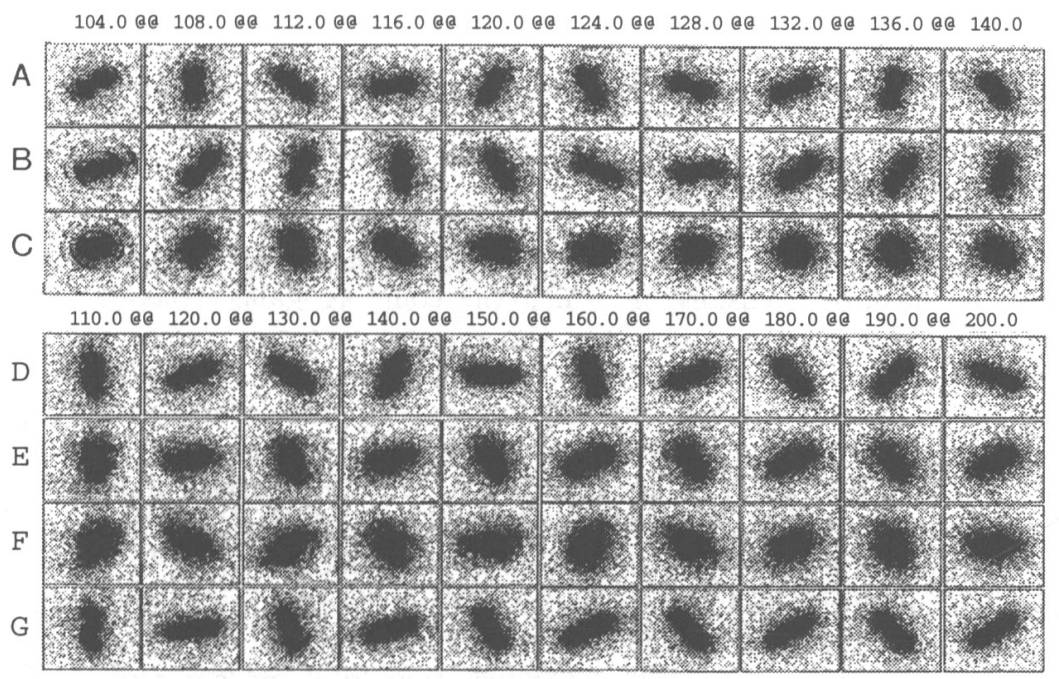

Figure 1. The evolution of the distribution of stars at several time steps. The length of each side of the squares corresponds to $6 a_{d}$. The numbers written on the top of squares indicate dynamical times. Direction of stars and bar pattern rotation is counter clockwise. Simulations $\mathrm{A}, \mathrm{B}$, and $\mathrm{C}$ are case (1), where combination of $|\vec{R}|$ and azimuthal angle of $\vec{R}$ are $(0,0),\left(0.5 a_{d}, 0\right)$ and $\left(0.5 a_{d}, \pi / 3\right)$, respectively. Simulations $\mathrm{D}, \mathrm{E}, \mathrm{F}$, and $\mathrm{G}$ correspond to case (2), where combinations of amplitude $A$ and mode $m$ are $(0,0),(0.01,1),(0.02,1)$, and $(0.02,2)$, respectively.

We performed 2 kinds of simulations for the disks after $t=100$ :

(1) Dynamical evolution of the disk after an instantaneous momentum change of stars using the impulse approximation for galaxy encounters.

The colliding galaxy is modeled by a Plummer sphere of mass $M_{c}=M_{t}$ and scale length $a_{c}$. This colliding galaxy passes through the position $\vec{R}$ of the target galaxy disk perpendicularly at the time $t=100$. The momentum change of disk stars at $\vec{r}$ is obtained analytically by the impulse approximation.

(2) Dynamical evolution of the disk after a finite amplitude potential perturbation.

A perturbing potential $\phi_{p}(r, \theta)$ is obtained by solving Poisson's equation with the perturbing density $\rho_{p}(r, \theta)$. The functional form of the perturbing density is artificial: $\rho_{p}(r, \theta)=A e^{i m \theta}\left\{k_{1}\left(r^{2}+a_{1}^{2}\right)^{-m_{1}}+k_{2}\left(r^{2}+a_{2}^{2}\right)^{-m_{2}}\right\}$, where $m_{1}=3$, $m_{2}=2, a_{1}=a_{2}=a_{d}$, and $k_{1}$ and $k_{2}$ are determined by $\int \vec{r} \rho_{p}(\vec{r}) d \vec{r}=\overrightarrow{0}$ and $2 \pi \int\left\{k_{1}\left(r^{2}+a_{1}^{2}\right)^{-m_{1}}+k_{2}\left(r^{2}+a_{2}^{2}\right)^{-m_{2}}\right\} r d r=\mathrm{M}_{t}$. 
The perturbing forces in the central regions of the disks in case (2) are very strong and artificial, so they represent an upper limit for interactions, but in case (1), they represent a lower limit for interactions.

\section{Results and Discussions}

Figure 1 shows results of the 2 kinds of simulations. Barred structures are destroyed by head-on collisions in which the colliding galaxy passes through at a somewhat displaced position from the galactic center along the bar minor axis and by one-armed perturbations of certain amplitude. But they are not destroyed by collisions in which the colliding galaxy passes through the center of the target galaxy or at a somewhat displaced position from the center along the bar's major axis. To draw definite results, we must survey much more in parameter space, but we can say as a preliminary result that barred structure can be destroyed in a dynamical time scale at the galactic center.

The reason why barred structure can be destroyed by such interactions or one-armed perturbations is as follows: One-armed perturbations can be considered as a superposition of parallel transformations of some parts of the disk, namely, if the central region of the galactic disk is pulled in one direction, then the outer region is pushed in the opposite direction. On the other hand, the gravitational field in the galactic center region is something like a "force free" field. So the central part of the barred structure can be displaced easily by a one-armed mode perturbation, and once displaced at a certain distance from the center, mixing by rotational motion around the galactic center occurs and the bar is destroyed.

\section{References}

Gerin, A., Combes, F., \& Athanassoula, E. 1990, A\&A, 230, 37

Noguchi, M. 1987, MNRAS, 228, 635 